Spring 4-25-2020

\title{
Animal-Assisted Therapy and Quality of Life in Patients with Dementia
}

Karissa D. Thomson

University of St. Augustine for Health Sciences

DOI: https://doi.org/10.46409/sr.CDZZ6449

Follow this and additional works at: https://soar.usa.edu/capstones

Part of the Neurology Commons, Occupational Therapy Commons, and the Psychiatry and Psychology Commons

\section{Recommended Citation}

Thomson, K. D. (2020). Animal-Assisted Therapy and Quality of Life in Patients with Dementia. [Doctoral project, University of St Augustine for Health Sciences]. SOAR @ USA: Student Capstone Projects Collection. https://doi.org/10.46409/sr.CDZZ6449

This Capstone is brought to you for free and open access by the Student Research at SOAR @ USA. It has been accepted for inclusion in Student Capstone Projects by an authorized administrator of SOAR @ USA. For more information, please contact soar@usa.edu, erobinson@usa.edu. 


\section{ANIMAL-ASSISTED THERAPY AND QUALITY OF LIFE IN PATIENTS WITH DEMENTIA}

by

Karissa D. Thomson

A Capstone Presented in Partial Fulfillment of the Requirement for the Degree of DOCTOR OF OCCUPATIONAL THERAPY University of St. Augustine for Health Sciences April, 2020 


\title{
ANIMAL-ASSISTED THERAPY AND QUALITY OF LIFE IN PATIENTS WITH DEMENTIA
}

by

\author{
Karissa D. Thomson \\ has been approved
}

April, 2020

APPROVED:

Kayla Collins, EdD, OTR/L, Doctoral Coordinator

Thais Petrocelli, OTD, OTR/L, Doctoral Coordinator

Mary Shotwell, PhD, OT/L, Program Director

ACCEPTED AND SIGNED:

Kayla Collins $\begin{aligned} & \text { Digitally signed by Kayla Collins } \\ & \text { Date: } 2020.07 .22 \text { 10:47:35 } \\ & -04^{\prime} 00^{\prime}\end{aligned}$

Kayla Collins, EdD, OTR/L, Doctoral Coordinator

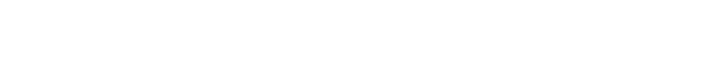

Thais Petrocelli, OTD, OTR/L, Doctoral Coordinator

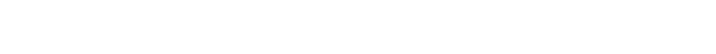

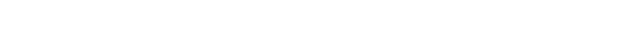

Mary Shotwell, PhD, OT/L, Program Director 
Table of Contents

$\begin{array}{lc}\text { Chapter } 1 & 6\end{array}$

$\begin{array}{ll}\text { Introduction } & 6\end{array}$

$\begin{array}{ll}\text { Background } & 7\end{array}$

Statement of the Problem 9

$\begin{array}{ll}\text { Purpose Statement } & 10\end{array}$

$\begin{array}{ll}\text { Project Rationale } & 10\end{array}$

Project Significance $\quad 11$

Definition of Terms $\quad 11$

$\begin{array}{ll}\text { Assumptions } & 12\end{array}$

$\begin{array}{ll}\text { Project Objectives } & 12\end{array}$

$\begin{array}{ll}\text { Conclusion } & 13\end{array}$

$\begin{array}{ll}\text { Chapter } 2 & 13\end{array}$

Literature Review 13

Why AAT Isn't Being Implemented More Often in Practice 16

OTs Role in Dementia Care 17

$\begin{array}{ll}\text { Dementia and Quality of Life } & 18\end{array}$

Impact of AAT on Patients with Dementia 18

OTs as Providers of AAT 19

Theory: Cognitive- Behavioral Frame of Reference 20

$\begin{array}{ll}\text { Conclusion } & 20\end{array}$

$\begin{array}{ll}\text { Chapter 3: Project Description } & 21\end{array}$

$\begin{array}{ll}\text { Introduction } & 21\end{array}$ 
$\begin{array}{ll}\text { Research Question } & 22\end{array}$

$\begin{array}{ll}\text { Design } & 22\end{array}$

$\begin{array}{ll}\text { Participants } & 23\end{array}$

$\begin{array}{ll}\text { Recruitment } & 24\end{array}$

$\begin{array}{ll}\text { Informed Consent } & 24\end{array}$

$\begin{array}{ll}\text { Setting } & 25\end{array}$

$\begin{array}{ll}\text { Materials } & 25\end{array}$

$\begin{array}{ll}\text { Procedure } & 26\end{array}$

$\begin{array}{ll}\text { Data Source } & 27\end{array}$

$\begin{array}{ll}\text { Data Collection } & 28\end{array}$

$\begin{array}{lr}\text { Data Analysis } & 29\end{array}$

$\begin{array}{ll}\text { Alignment with Objectives } & 30\end{array}$

$\begin{array}{ll}\text { Conceptual Framework } & 31\end{array}$

$\begin{array}{ll}\text { Timeline } & 32\end{array}$

$\begin{array}{ll}\text { Conclusion } & 33\end{array}$

Chapter 4: Results and Analysis 34

$\begin{array}{ll}\text { Introduction } & 34\end{array}$

Research Question and Hypothesis $\quad 34$

Analysis of the Data $\quad 35$

$\begin{array}{ll}\text { Summary } & 41\end{array}$

$\begin{array}{ll}\text { Chapter 5: Summary } & 42\end{array}$

$\begin{array}{ll}\text { Introduction } & 42\end{array}$

Summary of the Study's Results $\quad 42$ 
Limitations

Delimitations

Implications for the Project to the Literature

Implications for the Project to the Profession

Implications for the Project to the Population Served and Society

Conclusion

References 


\section{Chapter 1}

\section{Introduction}

Dementia is an umbrella term used to classify conditions that cause memory loss. The most common type of dementia is Alzheimer's disease (Centers for Disease Control and Prevention [CDC], 2018). A major risk factor for developing dementia is age, with people over 65 being at an advanced risk (Alzheimer's Association, 2019). According to the Alzheimer's Association (2019), 5.8 million Americans are currently living with Alzheimer's dementia. This number continues to rise each day, as every 65 seconds a new person develops dementia. By the year 2050, 14 million Americans are expected to be diagnosed with a form of dementia (Alzheimer's Association, 2019). As this disease becomes more prevalent, the interest in nonpharmaceutical interventions has grown in popularity. People with dementia living in a nursing home are $75 \%$ to $90 \%$ more likely to exhibit changes in their behavior as the disease progresses (Tournier, Vives, and Postal, 2017). People with dementia often experience "neuropsychiatric symptoms such as apathy, depression, agitation, and anxiety” (Tournier et al, 2017, p. 51). Animal-assisted therapy (AAT) is a potential non-pharmaceutical intervention shown to have positive impacts on depression and anxiety (Pope, Hunt, and Ellison, 2016). AAT is an intervention style involving interactions between the patient, therapy dog, and dog handler to produce therapeutic outcomes (Grubbs, Artese, Schmitt, Cormier, and Panton, 2016). Previous research has shown AAT has benefits associated with improved quality of life, improved mood, decreased loneliness, and increased social participation (Pope et al., 2016). However, studies have suggested further research needs to be conducted to apply this information to the entire population of dementia. 


\section{Background}

According to the Centers for Disease Control and Prevention [CDC] (2016), 47.8\% of individuals diagnosed with dementia reside in long-term care facilities. As the number of people diagnosed with dementia steadily increases, the need for long-term care facilities would be expected to increase proportionately (Smith, Buckwalter, Kang, Ellingrod, and Schultz, 2008). This means the demand for social and recreational activities to maintain function and quality of life will increase also. Although the elderly would prefer to age in their homes, this desired request is not attainable for those with advanced dementia (De Rooij, Luijkx, Declercq, and Schols, 2011). As the disease progresses, patients will experience increased confusion impacting their ability to independently care for themselves safely (De Rooij et al., 2011). Some challenges numerous residents face living in long-term care facilities are feelings of "isolation, rejection, loneliness, depression, hopelessness, boredom, and low self-esteem" (Fick, 1993, p.529). AAT is a goal-oriented intervention shown to have therapeutic effects associated with quality of life, social participation, and depression (Lundqvist, Carlsson, Sjödahl, Theodorsson, and Levin, 2017). A major goal of long-term facilities is making social integration activities available to maintain quality of life and well-being (Bernstein, Friedmann, and Malaspina, 2000). AAT has been used by long-term care facilities to increase social engagement and to promote a positive outlook on life (Bernstein et al., 2000).

However, there are limited studies investigating AAT's impact on social engagement and depression in people with dementia (Bernstein et al., 2000). Facilities face numerous challenges when trying to implement social activities for patients with dementia due to their decline in cognitive status and communication skills (Pope et al., 2016). Nevertheless, facilities have implemented social and recreational activities into patients' routines as they feel these are 
valuable to maintain current function and quality of life (Pope et al., 2016). AAT could serve as an holistic intervention for people with dementia as animals are "nonjudgmental, and they rely on body language more than humans do, which is especially valuable when working with patients with impaired language skills" (Tournier, Vives, and Postal, 2017, p. 51).

Providing additional evidence regarding the therapeutic effects of AAT offers a new technique for occupational therapists (OTs) to utilize with patients who have dementia. Research has shown patients with dementia respond positively to AAT engagement (Aarskog, Hunskär, and Bruvik, 2019). However, the results obtained from these studies have not produced statistically significant data due to small sample sizes and short-lasting benefits of AAT (Pope et al., 2016).

OTs play a role in the maintenance of function, quality of life, social participation/integration, and psychosocial factors. OTs are trained to assess, promote, maintain, and modify occupations or environments to increase functional performance (State Affairs Group, 2019). OTs facilitate functional performance using therapeutic use of self and purposeful interventions addressing activities of daily living (ADLs) and instrumental activities of daily living (IADLs) (AOTA, 2019). Combining OT and AAT into a session allows the therapist to work on several skills without patients being aware they are engaging in therapy. Some of these skills include fine motor coordination, range of motion, and social engagement $(\mathrm{Hu}, \mathrm{Zhang}$, Leng, Li, and Chen, 2018). These skills can be implemented by completing activities such as brushing, petting, feeding the dog, throwing a ball, opening food containers related to feeding the dog, and talking with the dog in addition to other residents involved in the group (Hu et al., 2018). 


\section{Statement of the Problem}

As the stages of dementia progress, the person's ability to live independently will decline due to impairments in cognitive status. The caregiver burden is significantly high during these stages as the care for a family member with dementia can cause financial distress, a decline in physical health, social isolation, and psychological problems (Brodaty and Donkin, 2009). These factors leave caregivers with no choice but to move their loved one into a long-term care facility. This transition from their lifelong home to a long-term care facility can have negative effects on quality of life and well-being. Patients have reported feelings of isolation, depression, and decreased quality of life (Pope et al., 2016). The use of AAT to help intervene with these feelings has produced positive effects; however, some studies suggest the effects are inadequate (Pope et al., 2016). The limited amount of statistically significant data regarding the use of AAT and its effects it has on people who have dementia has resulted in therapists not utilizing the intervention style as often. The standard treatment for psychological issues is through prescribing medications; however, recent studies have shown psychological medications can produce adverse effects including unexpected deaths in this population (Tournier et al., 2017). Interventions limiting the use of pharmaceutical drugs are of high interest for healthcare providers caring for this population and these individuals' family members (Tournier et al., 2017). AAT as a non-pharmaceutical alternative offers benefits that impact psychological factors such as depression and social engagement (Tournier et al., 2017). According to Tournier et al., this intervention style has produced positive effects related to psychological factors due to less physical and cognitive demands required to participate (2017). Studies indicate a need for nonpharmaceutical interventions such as AAT to be developed for improving social interaction and decreasing depression in individuals with dementia; however, limited evidence leaves the efficacy of AAT in need of further investigation (Tournier et al., 2017). 


\section{Purpose Statement}

The project titled "Animal-Assisted Therapy and Quality of Life in Patients with Dementia" will be conducted at a Central Florida skilled nursing facility. Isolation and rejection are common feelings patients with dementia often report experiencing after making the transition to a nursing home or long-term care facility (Fick, 1993). Studies have shown feelings of isolation and rejection impact the patient's psychological health (Pope et al., 2016). Patients with dementia experience an altered psychological status translating to depression, decreased social engagement, and a decrease in quality of life (Tournier et al., 2017). AAT has been shown to provide positive outcomes on psychological health for patients who have dementia (Sellers, 2006). However, the current research supporting the use of AAT in this population is limited due to small sample sizes and short duration of studies (Lundqvist et al., 2017; Sellers, 2006). Currently, psychological symptoms are treated with pharmaceutical interventions; however, medications have been shown to have negative effects on health (Nordgren and Engström, 2014). The demand for non-pharmaceutical interventions has grown in popularity to treat psychological problems in patients who have been diagnosed with dementia (Nordgren and Engström, 2014). The purpose of this capstone project is to investigate the impact AAT has on patients who have dementia through a quantitative study. This study will examine the effects AAT has on social engagement, depression, blood pressure, and how these factors impact their quality of life.

\section{Project Rationale}

OTs are trained to assess and facilitate functional performance in daily occupations to maintain independence in people with dementia (Smallfield, 2017). OTs primary focus of intervention for people with dementia include health promotion, remediation, modification, and maintenance (Smallfield, 2017). These skills can be utilized to treat the numerous psychological 
and physical health factors impacted by dementia. Previous studies have shown AAT and OT are highly associated with each other as they seek to accomplish similar goals (Cipriani et al., 2013). Cipriani et al. (2013) discovered very few studies conducted by the OT profession regarding AAT's impact on dementia. To bring awareness and promote the use of AAT in practice, Cipriani et al. suggested further research needs to be conducted by OTs.

\section{Project Significance}

AAT has been shown to provide positive benefits for patients who have dementia such as decreased depression, increased social engagement, and better quality of life (Bernstein et al., 2000). Research indicates patients spend most of their day alone in their room (Marx et al., 2010). Currently, psychological symptoms are treated with antipsychotic medications despite recommendations to try alternative methods before use (Nordgren and Engström, 2014; Aarskog, et al., 2019). The reason for this recommendation is due to the increased risk of strokes and adverse effects associated with the use of medications (Mendes, 2019). Non-pharmaceutical alternatives are gaining popularity in research (Tournier et al, 2017). A popular nonpharmaceutical intervention being researched currently is AAT (Tournier et al, 2017). While AAT has many positive benefits associated with its use the research base is limited due to small sample sizes, weak research designs, and short duration studies conducted (Pope et al., 2016).

\section{Definition of Terms}

- Animal-Assisted Therapy: "an intervention in which an animal is incorporated as an integral part of the treatment process, in order to promote an improvement in physical, psychosocial, and/or cognitive functioning of the person treated" (Sánchez-Valdeón et al., 2019, p.2) 
- Psychosocial factors: "psychological and social aspects of individual, groups and environments thought to be active in particular situations" (Psychology Wiki, 2019, para.1)

- Logic model: used to visually organize information and determine how the information collected will operate within the populations' needs (University of Minnesota, 2019).

\section{Assumptions}

This capstone project assumes the participants included in the study are interested in animals and want to engage with them as participation in this study is voluntary. The study assumes participants would like to decrease depression and increase social engagement to improve their quality of life.

\section{Project Objectives}

Learning objectives:

- Identify literature related to animal-assisted therapy and dementia care.

- Develop an experimental study to investigate the impact animal-assisted therapy has on social participation, depression, and blood pressure in relation to the quality of life of patients with dementia.

- To assess the logistics of developing a quantitative study to prepare for future research development.

Outcome objectives:

- Conduct a quantitative study on the impact of AAT on patients with dementia.

- Improve the social engagement of older adults with dementia using AAT as demonstrated by the participant's increased desire and participation in social activities. 
- Decrease depressive behaviors of people with dementia through AAT, as evidenced by the participant's increased rate of laughter, smiling, and spontaneously engaging with others.

- Analyze the effects AAT has on blood pressure, as evidenced by readings recorded pre and post sessions.

- Receive feedback from the site supervisor and evaluate the data collected from the sessions to determine the effectiveness of AAT for patients with dementia.

\section{Conclusion}

As the number of new diagnoses continue to rise daily, it is important to find alternative interventions that are cognitively stimulating and bring joy to this population. Nonpharmaceutical interventions are becoming popular for this population in research, as medications can cause harmful adverse effects. AAT has been shown to have a positive impact on behaviors which $75-90 \%$ of people living in a nursing home struggle to maintain (Tournier et al., 2017). Currently, the lack of statistically significant evidence from past research studies has hindered the use of AAT with people who have dementia living in a nursing home.

\section{Chapter 2}

\section{Literature Review}

Currently, 5.8 million people are living with a form of dementia, $50 \%$ of whom reside in a nursing home or long-term care facility (Alzheimer's Association, 2019). A report conducted by the Alzheimer's Association states, “the risk of Alzheimer's increases with age, and as baby boomers get older, the number of people developing the disease will rise to levels far beyond anything we've ever seen before" (Frantz, 2015, p.1). Unfortunately, there is no cure for dementia as it is a progressive disease (Hu et al., 2018). Due to the severity of the disease, it 
causes significant financial costs for families, society, and healthcare (Sánchez-Valdeón et al., 2019). It is estimated by $2040,25 \%$ of Medicare spending will be consumed caring for people who have been diagnosed with dementia (Frantz, 2015).

Dementia and Alzheimer's Disease are commonly associated with physical and cognitive impairments including neurobehavioral complications which adversely impact psychological health (Motomura, Yagi, and Ohyama, 2004; Tournier et al., 2017). Depression, agitation, and anxiety are exceedingly high for this population which negatively impacts the quality of life (Aarskog et al., 2019). Residents who live in a nursing home or long-term care facility have often reported feelings of isolation, rejection, and loneliness (Pope et al., 2016). Patients with dementia commonly experience "delusions, depression, apathy, irritability, anxiety, sleep disorders, and/or decline in social engagements" (Motomura et al., 2004, p. 40). It has been reported, patients living in a nursing home spend $22 \%$ of their time alone (Marx et al., 2010). Therefore, this population experiences adverse psychological conditions that may be negatively affected by the isolating environment of a long-term care facility.

Furthermore, approximately $80 \%$ of people diagnosed with dementia demonstrate aggressive and agitated behaviors (Nordgren and Engström, 2014). The primary treatment for adverse behaviors includes the use of "antidepressants, mood stabilizers, anxiolytics, hypnotics, and antipsychotics or cholinesterase inhibitors" (Nordgren and Engström, 2014, p. 31). The use of medications to treat psychological symptoms in patients who have dementia has created controversy (Aarskog, et al., 2019). Pharmaceutical interventions used to treat psychological symptoms are limited in usefulness and produce adverse side effects that could be harmful to this population (Aarskog et al., 2019). Adverse side effects and potentially harmful risks associated with pharmaceutical interventions have caused healthcare providers to investigate safer non- 
pharmaceutical alternatives for the treatment of behavioral and psychological problems ( $\mathrm{Hu}$ et al., 2018). Additionally, healthcare providers are recognizing the importance of cognitively stimulating activities that do not primarily focus on physical limitations to promote a better quality of life for this population (Williams and Jenkins, 2008).

Non-pharmaceutical interventions, such as AAT, have been shown to provide positive effects on socialization and to combat loneliness (Banks and Banks, 2002). AAT has been shown to be a promising example of a non-pharmaceutical intervention for patients who have dementia (Nordgren and Engström, 2014). AAT is defined as "an intervention in which an animal is incorporated as an integral part of the treatment process, in order to promote an improvement in physical, psychosocial, and/or cognitive functioning of the person treated" (Sánchez-Valdeón et al., 2019, p.2). The use of AAT as a form of treatment dates to the 1970s (Kanamori et al., 2001). AAT has been utilized to improve mental health by focusing on the human-animal relationship, which research has indicated improves independence and consequently improves the quality of life (Kanamori et al., 2001). A major focus of long-term care facilities is to reduce social isolation amongst patients who have dementia and AAT has been commonly utilized to engage patients in socialization (Berstein, Friedmann, and Malaspina, 2000).

The bond between a human and an animal, such as a dog, can have a positive impact on psychological and physiological factors (Aarskog et al., 2019). AAT interventions are delivered through human-animal interactions such as petting, brushing, playing, feeding, and walking the animal (Zafra-Tanaka, Pacheco-Barrios, Tellez, and Taype-Rondan, 2019). These interactions supported by use of AAT have shown promising results for psychiatric and cognitive conditions (Zafra-Tanka et al., 2019). Dogs are most used for AAT and demonstrate a friendly persona making them most favored for this type of intervention (Lundqvist et al., 2017). 
AAT has been shown to have positive effects on physiological systems such as the cardiovascular system, pain perception, and overall health (Hu et al., 2018). The release of oxytocin in the brain during interactions with an animal have been attributed to the positive effects related to physiological and psychological factors (Beetz, Uvnäs-Moberg, Julius, and Kotrschal, 2012). When the oxytocinergic system is activated in the brain, hormonal changes have been shown to have a positive impact on psychological factors such as increased social interaction, empathy, positive mood, and decreased aggression (Beetz et al., 2012). The activation of the oxytocinergic system caused by animal interaction has shown to have positive effects on the physiological system such as decreased blood pressure, heart rate, and changes in hormonal levels such as cortisol, epinephrine, and norepinephrine insinuating diminished stress levels (Beetz et al., 2012). Due to the physiological benefits of AAT, it has been utilized to maintain, promote, and restore physical, psychological, and sociological function in those with an altered cognitive status (Tournier et al., 2017).

\section{Why AAT Isn’t Being Implemented More Often in Practice}

AAT research studies have increased in demand for not only healthcare professionals but also the general public (López-Cepero Borrego et al., 2014). However, the current evidence regarding the use of AAT is weak due to small sample sizes (Lundqvist et al., 2017). Although small sample sizes are a major limitation for the generalizability of AAT use, there have been positive benefits reported from previous studies conducted (Williams and Jenkins, 2008). Most studies have shown positive benefits for patients who have dementia, but how AAT impacts psychological factors is unclear (Bernabei et al., 2013). Williams and Jenkins (2008) found a lack of empirical evidence in the literature regarding the use of AAT and a significant amount of studies indicate further research is essential. Studies investigating the appropriate duration and 
frequency of AAT to provide therapeutic effects require more research before being applied to the population of dementia (Bernanei et al., 2013). Klimova, Toman, and Kuca (2019) suggested the need for further research studies focusing on how AAT impacts cognitive functions of people with dementia. Additionally, weak research designs limit the use of AAT in practice and researchers have concluded more quantitative randomized double-blind controlled studies should be conducted to provide statistically significant data (Chur-Haansen, Stern, and Winefield, 2010).

\section{OTs Role in Dementia Care}

OTs concentrate on occupational performance to maintain current function as it is significantly impacted by the progressive nature of dementia (Smallfield, 2017). OTs analyze how the advancement of the disease has impacted the patient's occupational performance using an activity analysis (Smallfield, 2017). To treat patients who have dementia, OTs primarily focus on four intervention factors: health promotion, remediation, maintenance, and modification (Smallfield, 2017). The core of OT for patients with dementia includes the promotion of safety, maintain occupational engagement, and increase the quality of life (American Occupational Therapy Association [AOTA], 2019). However, to obtain these goals, OTs must consider the stage of dementia and the potential outcome to be obtained from the intervention (American Occupational Therapy Association [AOTA], 2019). Specific outcomes of OT include maintenance of current function in specific ADLs and IADLs. The OT profession is recommended due to their knowledge base to provide interventions for physical and psychosocial factors to promote a better quality of life for patients with dementia (Gately and Trudeau, 2017). 


\section{Dementia and Quality of Life}

As the severity of dementia continues to progress, its negative impact on cognition increases causing a decline in cognitive function. Due to the progressive nature of dementia, the ability for people with later stages of dementia to live independently at home safely becomes difficult and is ultimately not possible (De Rooij et al., 2011). Dementia causes people to experience a physical decline in everyday tasks, memory loss, and negatively impacts moods and behavior (Bauer et al., 2018). In the beginning stages of dementia, the patient can obtain care while at home but as the disease continues to progress 24-hour care provided by a medical professional will be required (Makai, Beckebans, Van Exel, and Brouwer, 2014). Quality of life and functional ability have been directly linked to each other (Bauer et al, 2018). As functional ability declines, the patient's overall quality of life has been shown to decline (Bauer et al., 2018). This could ultimately lead to psychological problems such as depression, social isolation, and anxiety (Bauer et al., 2018). Of the psychological symptoms reported, depression has been associated with a greater correlation to quality of life than any other psychological symptom (Wetzels, Zuidema, Jonghe, Verhey, and Koopmans, 2010). Lewy body disease, a form of dementia, has been linked to a lower quality of life as compared to those with Alzheimer's disease (Wetzels et al., 2010). Thus, the type of dementia should be taken into consideration when examining the quality of life in patients who have dementia (Wetzels et al., 2010).

\section{Impact of AAT on Patients with Dementia}

AAT has been shown to provide positive benefits for patients who have dementia including reductions in agitation, lethargy, aggression, blood pressure, and increased relaxation (Williams and Jenkins, 2008). Observations made during studies have noted increased calmness and relaxed behaviors which have an impact on psychological disorders such as depression and 
anxiety (Klimova et al., 2019). Self-control and socialization have been shown to increase with the use of AAT (Bernabei et al., 2013). AAT has been shown to be supportive for the dementia population as animals are nonjudgmental and will not have a negative reaction to repeated phrases and stories, thus allowing patients to engage in social activities (Marx et al., 2010). The presence of an animal has been associated with decreased emotional outbursts and loud behaviors within the unit promoting a quiet environment (Marx et al., 2010). Thus, AAT can be used as an additional intervention to help alleviate common psychological comorbidities and increase social participation.

\section{OTs as providers of AAT}

AAT can be utilized by OTs to address cognition, range of motion, strength, and balance (Velde, Cipriani, and Fisher, 2005). OTs have additionally used AAT to promote motor skills, wheelchair skills, activity participation, attention to tasks, and completion of simple tasks (Muñoz Lasa and Franchignoni, 2008). OTs have noted increased motivation to participate in therapy when an animal is present, especially for those who normally refuse therapy (Velde et al., 2005). OTs have reported they can provide therapy for a longer duration when an animal is involved to reach greater accomplishments toward goals (Velde et al., 2005). Previous OTs have observed improvements in range of motion, sensory modulation and interpretation, and increased activity tolerance during sessions (Velde et al., 2005). OTs refer to AAT as a modality utilized to promote/create, establish/restore, maintain, modify, and prevent specific factors (Velde et al., 2005). An example of this would be to focus on feeding a therapy dog and discussing the importance of eating properly with a patient who may be refusing to eat. This type of intervention could spark an interest in the patient to participate with the OT to establish his/her nutritional routine. 


\section{Theory: Cognitive Behavioral Frame of Reference}

The cognitive-behavioral frame of reference focuses on psychological barriers limiting a person's performance in occupation (Cole and Tufano, 2008). It discusses how emotions can have an impact on occupational performance throughout the lifespan. The cognitive-behavioral theory is widely used in behavioral settings to address motivation and emotional problems (Cole and Tufano, 2008). In this frame of reference, cognition is heavily examined through evaluation and interventions as it impacts occupational performance (Cole and Tufano, 2008). Cognitivebehavioral theory can be used to address cognitive distortions often associated with depression. For instance, a patient who is having feelings of rejection due to their family never coming to visit may limit their social engagement with others in fear of being rejected by them. This cognitive distortion the patient believes in leads them to seclude themselves from others and remain in their room causing depression, social isolation, and decreased quality of life. AAT and cognitive-behavioral theory could be paired together to implement positive thinking by allowing them to interact with a therapy dog and other residents during the AAT sessions.

\section{Conclusion}

Based on current evidence found in the literature, one can infer AAT can have positive effects on patients living in a nursing home. AAT, as an alternative intervention, can be utilized to increase social engagement, decrease depression, and increase the quality of life. Currently, the gaps in the literature related to the use of AAT for patients who have dementia are hindering its usage. Further quantitative studies should be conducted to support the use of AAT for patients with dementia. The increase in literature could provide additional knowledge regarding the use of AAT. OTs implementing AAT into practice could provide this population with increased social participation, psychological, and physiological benefits leading to improved quality of life. 


\section{Chapter 3: Project Description}

\section{Introduction}

The purpose of this capstone project is to conduct a quantitative research study to investigate the impact AAT has on quality of life in the dementia population. This study will examine the effects AAT has on depression, social engagement, and blood pressure. It will then examine how these factors impact the quality of life of patients who have dementia living in a nursing home.

The elderly living in a nursing home are 3 to 4 times more likely to develop depressed behaviors as compared to the elderly who reside in a private residence (Zhao et al., 2018). Loneliness is a risk factor for depression and is often associated with social isolation (Zhao et al., 2018). Agitation and anxiety-driven behaviors are elevated in the dementia population (Aarskog et al., 2019). Psychological behaviors in patients with dementia can intensify based on the severity of their dementia, environmental factors, unmet needs, and their overall health (De Bellis, Bradley, Xiao, Belan, and Wallace, 2017). To help alleviate abnormal psychological behaviors, antipsychotic medications are primarily used regardless of recommendations to utilize non-pharmaceutical interventions first (De Bellis et al., 2017). It has been found that antipsychotic medications are inappropriate for two-thirds of people with dementia (Mendes, 2019). Antipsychotic medications are often associated with adverse events such as increased risk for stroke, cerebrovascular events, and mortality (Mendes, 2019).

The use of non-pharmaceutical interventions for the dementia population is recommended (De Bellis et al., 2017). A commonly known non-pharmaceutical intervention is AAT. AAT has been associated with positive results for the dementia population. AAT has shown improvements in depression, social engagement, blood pressure, and agitation (Hu et al., 
2018; Klimova et al., 2019). However, current studies suggest AAT is in the early research development stages (Wood, Fields, Rose, and McLure, 2017).

Recent studies have examined the effects of AAT on psychological behaviors such as depression and agitation (Hu et al., 2018). Studies have concluded AAT offers positive results for social integration, blood pressure, and depression (William and Jenkins, 2008; Bernabei et al., 2013). However, each study suggests further research is required to generalize this data to the entire dementia population (Hu et al., 2018). A study conducted by Cipriani et al. (2013) found a strong correlation between OT and AAT, however, there are limited studies involving OTs investigating AAT. Cipriani et al. (2013) stressed the importance of OTs involvement in conducting studies regarding AAT use to strengthen the research base. Other studies have suggested small sample sizes, short duration, and limited quantitative studies conducted have impacted the ability to generalize the use of AAT to the entire dementia population (Klimova et al., 2019: Hu et al., 2018).

\section{Research question}

In patients diagnosed with dementia who reside in a nursing home, how does AAT impact depressive behaviors, social interactions, and blood pressure? How do these factors once impacted by AAT contribute to this patient group's overall quality of life?

\section{Design}

This study was conducted as a quantitative research study with a pre-test, post-test single group design to collect data on the effects AAT had on blood pressure, depression, social integration, and its impact on the quality of life (Cole and Tufano, 2008). Cipriani et al. (2013) suggested higher quality research designs and studies conducted by the OT profession for the use of AAT should be performed. Other studies have recommended there needs to be an expansion 
of quantitative randomized studies conducted to widen the evidence-base for AAT (Chur-Hansen et al., 2010). Based on these current needs and recommendations from the literature, this study was conducted as a quantitative research study. Previous literature revealed meeting once a week for one hour was typical for interventions performed in nursing homes (Tournier et al., 2017). This study took place one hour once a week for six weeks. The participants were divided into two groups: mild dementia as group one and moderate dementia as group two.

\section{Participants}

People over the age of 65 are at higher risk for the development of dementia (Alzheimer's Association, 2019). The risk of developing dementia over the age of 65 doubles every 5 years (Alzheimer's Society, 2016). Therefore, the participants included in this study were 65 years and older. Nineteen residents living in a nursing home were randomly selected to participate in the study. Informed consents were obtained from each resident's power of attorney prior to interaction with the resident. After discussing the study with each resident, thirteen were interested in participating. Two residents passed away prior to the start of the study and four residents decided they did not want to participate; therefore, seven total participants were included in the study. Participants were divided into two groups based upon the severity of their dementia. The determination of which group the participant was placed in was chosen based upon their score obtained on the Mini-Mental Status Examination (MMSE).

Inclusion criteria: over 65 years old, interested in animals, diagnosed with mild or moderate dementia,

Exclusion criteria: under 65 years old, allergic to animals, fearful of animals, not interested in animals, scored a 12 or below on the MMSE indicating severe dementia 


\section{Recruitment}

Due to increased confusion, the researcher found it more appropriate to obtain a list from the nursing manager for patients who have been diagnosed with dementia. The researcher then called each resident's power of attorney (POA) to explain the study and obtain signed informed consent forms prior to discussing the study with the resident. Each resident was then interviewed to determine their interest in participating in the study. During the initial interview, the following questions were asked to determine if the resident was a candidate to participate in the study: 1 . Have you ever owned an animal? 2. Are you interested in animals? 3. Do you have any allergies to animals such as a dog? 4. Do you like dogs? 5. Do you have any fear of dogs? 6. Would you like to participate in a study involving interactions with a therapy dog? If the participant answered "yes" to these questions, then their name and room number was inputted on a graph to come back and conduct further assessments with.

\section{Informed Consent}

Each of the participants living in the nursing home had active POAs listed on file. Thus, informed consents were obtained from each of the participant's POAs. The procedure and core of the study were explained to each of the POAs before obtaining informed consent. Electronic informed consents were sent via email to each POA who were unable to meet in person to obtain signatures. Informed consents were uploaded to a password-protected file on the researcher's laptop for documentation purposes. The POAs and participants were made aware their participation in the study was completely voluntary and they had the option to withdraw at any time without penalty. 


\section{Setting}

The study took place at a Central Florida skilled nursing facility. The study was conducted in a private activity room located within the memory care unit to reduce stress levels and environmental stimulation.

\section{Materials}

One certified therapy dog and dog handler who volunteered to participate in the study were utilized. The therapy dog and dog handler were present at each session over the 6 weeks. The therapy dog utilized in this study was a 20-pound Australian Shepard and Poodle mix.

A private room with a door located in the memory care unit was reserved to conduct the interactions between the therapy dog and participants. The use of a private room allowed the researcher to control for environmental overstimulation, reduce stress, and promote a quiet environment. Additionally, the utilization of a private room kept the residents who were not associated with the study separated to control for interruptions and distractions. The facility supplied chairs with armrests to reduce fatigue during the hour session for each group of participants, however, most residents required a wheelchair for mobility.

Three assessments were used to collect data regarding the participant's quality of life, depression, and cognition. The Mini Mental State Examination (MMSE) was used to assess the cognitive status of each participant to determine which group the participant would be placed in. The Beck's Depression Inventory (BDI) was used to measure the participant's current level of depression before AAT and again at the end of the study for comparison purposes. The World Health Organization Quality of Life BREF (WHOQOL-BREF) was used to quantify participants' satisfaction with their quality of life, pre, and post-intervention. 


\section{Procedure}

The researcher presented to the Institutional Review Board (IRB) committee the study with support as to why it needs to be conducted. The IRB committee ensures the safety and protection of human subjects' rights who are participating in a study. Information regarding how it would be conducted, safety measures, what activities would take place were all included in the documents submitted to the IRB committee. A convened IRB was completed due to the study being conducted with a vulnerable population.

The researcher obtained from the nursing manager a list of patients diagnosed with dementia who reside in the memory care unit. The examination of each participant's medical file was conducted for inclusion and exclusion criteria before interviews. The researcher then conducted individual interviews with each potential participant to determine if they were a candidate for the study.

Pre-assessments were conducted after informed consents were obtained from each of the participant's POA. The questionnaires were administered before the start of the AAT sessions. The MMSE, BDI, and WHOQOL-BREF were administered individually in a quiet, private room. Participants were offered 5 to 10 -minute breaks every 15 minutes while conducting the questionnaires to limit fatigue. The researcher then scored the MMSE to determine placement in the mild or moderate dementia group. Participants who scored between 20-24 were placed in the mild group while scores between 13-19 qualified for the moderate group. Blood pressure readings were obtained at each session before and after interactions with the therapy dog.

The AAT sessions were conducted in a quiet, private room located in the memory care unit. The mild dementia group attended the AAT session first, followed by the moderate dementia group. Each session lasted for one hour once a week for six weeks. During the AAT 
session, participants were provided the opportunity to pet, hug, brush, and talk to the therapy dog. The participants were observed for specific behaviors during their interaction with the therapy dog. These behaviors included smiling, laughter, flat affect, withdrawal, and engagement in social interactions with others.

BDI and WHOQOL-BREF were administered after the last session. A study conducted by Banks and Banks (2002) found administering pre and post-tests strengthened statistical significance by comparing change throughout the study. The scores from the pre-session will be compared to the post-session to determine the impact of AAT.

\section{Data Source}

According to the Shirley Ryan Ability Lab (2014), the WHOQOL-BREF is a highly recommended assessment when there is a time constraint or limiting fatigue is a concern. Internal consistency for the four domains: physical, psychological, social relationship, and the environment was rated as excellent for the use with patients who have dementia (Shirley Ryan Ability Lab, 2014). According to the World Health Organization (2019), the WHOQOL-BREF demonstrates "good discriminant validity, content validity, and test-retest reliability". A study conducted by Lucas-Carrasco, Skevington, Gómez-Benito, Rejas, and March (2011) determined patients diagnosed with dementia who are in the early stages can provide reliable and valid information regarding their quality of life. Additionally, they used this assessment on patients who were diagnosed with mild to moderate stages of dementia (Carrasco et al., 2011).

According to a study conducted by Tombaugh and McIntyre (1992) reliability and construct validity of the MMSE were satisfactory while criterion validity was highly sensitive for moderate to severe cognitive impairment. The study suggested the determination of dementia should be diagnosed with other clinical assessments; however, the MMSE serves as a quick 
screening tool to determine if deficits are present (Tombaugh and McIntyre, 1992). The MMSE is a popular screening test utilized to determine if there are cognitive deficits present in older adults (Baek, Kim, Park, and Kim, 2016).

The BDI is highly used by research and clinical personnel to determine the level of depression (García- Batista, Guerra-Pena, Cano-Vindel, Herrera-Martínez, and Medrano, 2018). The BDI has high concurrent validity and high construct validity for the general population (Statistical Solutions, 2019). Previous research indicated the BDI had a one-week test-retest reliability of 0.93 (Statistical Solutions, 2019). However, the BDI is not commonly used in the dementia population. According to Wagle, Ho, Wagle, and Berrios (2000), the use of BDI has not been fully evaluated for the use with patients who have dementia. This screening will not be used to diagnosis patients with depression but will be used to monitor depression preintervention and post-intervention.

\section{Data Collection}

The MMSE was used before the start of the AAT sessions to determine which group the participants were placed in. This assessment measured the participant's cognitive status and the severity of their dementia. Participants who scored between 20-24 were classified as mild dementia and placed in group one. Participants who scored between 13-19 were classified as moderate dementia and placed in group two.

The BDI was utilized to measure the participant's current level of depression before and after the administration of AAT. One question pertaining to sex life was excluded from use as the researcher determined it was inappropriate to ask in this setting. Additionally, the researcher observed interactions with the therapy dog with specific attention to laughter, smiling, and 
initiation of social engagement. This was observed to determine how AAT impacts participants' social engagement with others and if there is an increase in these factors.

The WHOQOL-BREF was used to determine the participant's current outlook on their quality of life before the start of the study. One question regarding sex life was excluded as the researcher determined it was not essential to ask in this setting. The assessment was then administered again after the 6 sessions to determine if the participant's quality of life was impacted by AAT. The assessment was administered the following day after the last session to compare pre and post scores.

Previous research has suggested AAT has positive effects on blood pressure, therefore, blood pressure was examined in this study (Klimova et al., 2019). The history of blood pressure medication was reviewed to determine if patients are currently taking medication to regulate their blood pressure. The researcher and site supervisor, who is a licensed OT, conducted blood pressure readings on each participant before the start of the session and at the end of each session. Blood pressure readings were conducted at the same time at each session. For group one (mild dementia group), blood pressure readings were taken at 1:00 pm and again at 2:00 pm. For group two (moderate dementia group), blood pressure readings were taken at 2:00pm and 3:00pm.

\section{Data Analysis}

To compare data collected during the study, the Wilcoxon signed- rank test was utilized to determine if the null hypothesis should be accepted or rejected. This test was chosen due to the small sample size which affected a normal distribution of sample means. Pre and posttest data were analyzed using the Wilcoxon signed-rank test for the WHOQOL- BREF, BDI, and blood pressure recordings. Only the initial blood pressure reading, and the last blood pressure reading 
were used for comparison in the Wilcoxon signed-rank test. The additional blood pressure readings were performed as precautionary measures to monitor the patient's health during the intervention.

\section{Alignment with Objectives}

The objectives for this capstone project are as follows: conduct a quantitative study on the impact of AAT on patients with dementia, improve the social engagement of older adults with dementia using AAT as demonstrated by the participant's increased desire and participation in social activities, decrease depression behaviors of people with dementia through AAT, as evidenced by the participant's increased rate of laughter, smiling, and spontaneously engaging with others, analyze the effects AAT had on blood pressure, as evidenced by readings recorded pre and post sessions, and receive feedback from site supervisor and evaluate the data collected from the sessions to determine the effectiveness of AAT for patients with dementia.

The methods section follows these objectives as it addresses the need for quantitative studies in addition to studies conducted by the OT profession regarding AAT (Cipriani et al., 2013). The study addressed social engagement and the impact AAT had on patients with dementia. It examined how AAT impacted depression by observing increased laughter, smiling, and any other positive behaviors witnessed during the sessions. Additionally, the results from the pre and post scores on the BDI were compared to determine any effect AAT had on depression. Blood pressure recordings were analyzed to determine if there were any decreases in blood pressure with the presence of a therapy dog. Feedback from the site supervisor was taken into consideration to revise the study to make it stronger for future research studies. All data collected during the study was analyzed and results were explained via a poster presentation presented at scholarship day at the researcher's home university. 


\section{Conceptual Framework}

Logic models are used to organize information and determine how the information collected will operate within the population's needs (University of Minnesota, 2019). Logic models address short-term, intermediate, and long-term goals developed by a company (University of Minnesota, 2019). A logic model provides a visual representation of the relationship between what is needed in the community and the plan to meet the need (Centers for Disease Control and Prevention [CDC], 2018). The components of a logic model are as follows inputs, activities, and outcomes (Centers for Disease Control and Prevention [CDC], 2018).

The inputs are factors put into the program or things invested in the program (Centers for Disease Control and Prevention [CDC], 2003). Printing of assessment materials and informed consents were the only costs associated with this study. The skilled nursing facility is providing materials needed to conduct the study such as a reserved private room, chairs, and a blood pressure machine. A licensed OT working at the facility was available to supervise during the duration of the study. A certified therapy dog and trained dog handler volunteered to provide services for the study.

Activities are what took place during the study to produce desired outcomes (Centers for Disease Control and Prevention [CDC], 2003). Assessments were administered before the start of the study and post-study to compare results. Blood pressure readings were collected for each participant. During the implementation process of AAT, participants interacted with a certified therapy dog. Participants had the option to choose how they would like to interact with the therapy dog. Options for interaction included playing, petting, brushing, lap sitting, and talking with the dog. The researcher collected data regarding laughter, withdrawal, flat affect, maintain interest, smiling, and initiation of social engagement with others included in their group. 
Outputs are defined as "direct, tangible results of activities-what we get" (Centers for Disease Control and Prevention [CDC], 2003, p. 2). The observations from the interactions with the therapy dog served as outputs of the study. It can be assumed observations of laughter, smiling, and interactions with others increased social participation. The information gathered from pre- and post-assessments served as tangible data from the study.

"Outcomes are the desired results of the program- what we achieve" (Centers for Disease Control and Prevention [CDC], 2003, p. 2). Short-term outcomes of the study included increased social engagement, laughter, and smiling. Intermediate outcomes of the study included a decrease in depression levels and an improved outlook on quality of life. Long-term effects of AAT's impact on patients with dementia will not be examined in this study. This study will serve as an example to present to other OTs the importance of their involvement in AAT research. It will serve as an example to develop a longer study including a larger geographical location to increase the generalizability of AAT for use with dementia.

\section{Timeline}

During the first 4 weeks, preparatory tasks were conducted to plan for the implementation of the therapy dog with participants included in the study. Week 1 consisted of the researcher obtaining a list of residents who have been diagnosed with dementia. Additionally, week 1 included the researcher beginning to call POAs to explain the study and obtain informed consent forms. During week 2 the researcher reviewed medical records for inclusion and exclusion criteria of each resident that a signed informed consent had been received for. Week 2 also consisted of the researcher interviewing potential participants to determine if they would be a candidate for the study. Additionally, week 2 consisted of the researcher following up with POAs who either did not answer the phone or had not returned the form. Week 3 consisted of the 
researcher administering assessments to each resident whose informed consents had been obtained for. At the end of week 3 and the duration of week 4, assessments were administered to all residents whose POA had approved for them to participate. All information was then uploaded to a password-protected file on the researcher's password-protected laptop.

During the next 6 weeks, the AAT sessions were conducted. Over the 6 weeks, observations were obtained regarding behaviors observed such as smiling, laughing, withdraw, flat affect, and initiation of social interactions. This data was not counted reliably and was only used as observations made from the interactions. The goal was to determine if the participants had any reaction to the dog. Additionally, blood pressure readings were obtained at each session over the 6 weeks.

The last 4 weeks consisted of the researcher obtaining feedback from the site supervisor regarding the logistics of the study. The researcher analyzed all data collected during the study. The data collected was then compared using the Wilcoxon signed-rank test to determine the significance of the information collected in the study. This information was then formulated into a paper and a poster presentation to discuss the outcomes obtained during the duration of the study. The poster presentation was presented at scholarship day at the researcher's home university regarding the impact AAT had on patients who have dementia and their quality of life.

\section{Conclusion}

Information collected from this study will aid in bridging the gap between OT and AAT as evidence is limited for this type of intervention. The data collected will be used as evidence to present to other facilities who care for patients with dementia. This includes patients living in long-term care facilities, nursing homes, adult day programs, and specialized homes for patients who have dementia. The presentation will provide other facilities with an example of how to 
conduct research in this area and advocate for the importance of the OT profession getting involved. Promoting other OTs to get involved in AAT research will help to build the evidence base for this intervention and help to generalize data to the dementia population. Future goals of this capstone project are to conduct a larger study including a larger geographical location to strengthen the evidence base.

\section{Chapter 4: Results and Analysis}

\section{Introduction}

This study concluded with seven participants completing the six AAT sessions. Originally, the study had thirteen participants who agreed to attend, however, two participants passed away, and four refused to attend sessions. Of the seven participants who attended sessions, two participants (7629 and 2194) missed one session during the 6 weeks.

The data for this study was collected using the WHOQOL-BREF, blood pressure recordings, and clinical observations. The results were analyzed using the Wilcoxon SignedRank test to determine the significance of data obtained. Clinical observations related to withdrawal, laughter, smiling, and any other behaviors were recorded during each session.

\section{Research Question and Hypothesis}

The research question for this study was as follows: In patients diagnosed with dementia who reside in a nursing home, how does AAT impact depressive behaviors, social interactions, and blood pressure? How do these factors once impacted by AAT contribute to this patient group's overall quality of life?

The hypothesis for this study was the following: Animal-assisted therapy will improve depressive behaviors, social interactions, and blood pressure resulting in an improved quality of life for people who have dementia living in a nursing home. 


\section{Analysis of the Data}

In table 1, the results from the MMSE were used to determine the severity of cognitive decline in participants with dementia. The participants were divided into two groups associated with mild cognitive decline and moderate cognitive decline.

Table 1

MMSE Results for Cognition and Placement in Group

\begin{tabular}{cccc}
\hline Participant & Score & Severity of Cognition & Group Placement \\
\hline 4689 & 22 & Mild & Group 1 \\
1045 & 20 & Mild & Group 1 \\
6932 & 16 & Moderate & Group 2 \\
7629 & 14 & Moderate & Group 2 \\
7936 & 15 & Moderate & Group 2 \\
7828 & 17 & Moderate & Group 2 \\
2194 & 14 & Moderate & Group 2 \\
\hline
\end{tabular}

In table 2, the Wilcoxon signed-rank test was used to analyze the results from pre and post AAT scores on the BDI. One question on the BDI regarding sex life was excluded from the study due to the researcher determining this was an inappropriate question to ask in the nursing home setting. When depressive behaviors were measured using the BDI, the results were mixed for both mild and moderate groups. Participant 4689 had a decrease in depressive behaviors following the six sessions, while participant 1045 had an increase, however, both post AAT scores were within normal range. Participants 6932, 7629, and 7828 had a decrease in depressive behaviors from their pre-AAT score. Participant 2194 had an increase in depressive behaviors from 11 to 18 indicating borderline clinical depression. During the post questionnaire, this participant was having a rough day which could have impacted the score.

The critical value is determined by the sample size of this study and is classified as 2 . The test statistic was then compared to the critical value to determine if the null hypothesis should be rejected or accepted. The test statistic for the BDI was 8 , which means it was greater than the 
critical value. When the test statistic is greater than the critical value then the null hypothesis is accepted. The null hypothesis is as follows: AAT did not impose any impact on depressive behaviors in patients who have dementia living in a nursing home. The data collected is assumed to be skewed as the sample size was significantly small and was not indicative of a normal distribution of data.

However, individual scores on the BDI assessing depression did decrease for most of the participants, although not enough to show significance. This could be due to the use of selfreport questionnaires for participants with a cognitive decline. It can be assumed people with a cognitive decline have difficulty understanding the question being asked or being unable to recall how they felt over the past two weeks which leading to an inaccurate representation of how they feel. Also, depending on the day the participant was having at the time of the post questionnaire could have had an impact on their score.

Table 2

BDI Results for Comparison of Depressive Behaviors Pre/Post AAT

\begin{tabular}{|c|c|c|c|c|c|c|c|}
\hline Participant & Pre & Post & Difference & Positive & Diff & Rank & Signed Rank \\
\hline 4689 & 14 & 5 & 9 & 1 & 9 & 6 & 6 \\
\hline 1045 & 0 & 3 & -3 & -1 & 3 & 2 & -2 \\
\hline 6932 & 11 & 6 & 5 & 1 & 5 & 3 & 3 \\
\hline 7629 & 16 & 11 & 5 & 1 & 5 & 3 & 3 \\
\hline 7936 & 4 & 5 & -1 & -1 & 1 & 1 & -1 \\
\hline 7828 & 23 & 6 & 17 & 1 & 17 & 7 & 7 \\
\hline 2194 & 10 & 18 & -8 & -1 & 8 & 5 & -5 \\
\hline
\end{tabular}

The WHOQOL-BREF was broken down into 4 different domains and each domain was scored individually based upon the participants raw scores. The four domains of the WHOQOLBREF includes (1) Physical Health, (2) Psychological, (3) Social Relationship, and (4) Environment. Higher post AAT scores are associated with higher QOL while lower scores are 
associated with lower QOL. Tables 3, 4, 5, and 6 correlates to the WHOQOL-BREF. One question related to sex life was excluded from the study as the researcher determined this was an inappropriate question to ask in the nursing home setting. Repeated Wilcoxon signed-rank tests were administered for each domain in the WHOQOL-BREF. All domain test statistic scores were higher than the critical value, therefore, the null hypothesis was accepted, and the alternate hypothesis was rejected.

For table 3 domain 1: physical health, five participants did not have improved QOL after the six sessions of AAT. Participant 7629 did not show any changes in their QOL from pre to post AAT sessions as their score remained the same. Participant 7828 scored a 31 on the post questionnaire indicating only a small change in QOL from a pre score of 30. This could be due to the participant having a good day or something influencing their answers but does not produce significance.

For table 4 domain 2: psychological, two participants (7639 and 7828) had an increase in QOL based upon their post AAT scores. All other participants scores decreased after the six sessions of AAT. It can be assumed the understanding of questions or recalling how they have felt over the past two weeks had an influence over their answers. It can also be assumed some participants may have given answers based upon how they felt in that moment when the questionnaire was administered.

For table 5 domain 3: social relationship, five participants had a decrease in post AAT scores while one had an increase, and another had no change. Participant 7828 had an increase in their post score which is indicative of an increase in QOL based upon their score. Participant 2194 had no change in their pre and post scores. Many reported they had no friends who came to visit them in the nursing home or had not made friends with others within the nursing home. The 
lack of personal relationships could have had a major impact on their scores as they have become secluded from society resulting in lost friendships when transitioning to a nursing home.

For table 6 domain 4: environment, QOL scores decreased for five participants, increased for one participant, and remained the same for another. Participant 7828 was the only participant in the study to increase their QOL in each domain of the WHOQOL- BREF. It can be assumed the interaction with the therapy dog and other participants influenced this participant's score of on the post AAT questionnaire. Although one participant showed positive results, the data collected does not produce statistically significant data based upon the Wilcoxon signed- rank test. It is assumed a larger sample size would have been more beneficial to produce significant data for the self-report questionnaire used in this study.

Table 3

WHOQOL-BREF Domain 1: Physical Health Raw Scores for Comparison of Quality of Life Pre/Post AAT

\begin{tabular}{cccccccc}
\hline Participant & Pre & Post & Difference & Positive & Diff & Rank & Signed Rank \\
\hline 4689 & 25 & 24 & 1 & 1 & 1 & 2.5 & 2.5 \\
1045 & 30 & 26 & 4 & 1 & 4 & 4.5 & 4.5 \\
6932 & 27 & 25 & 2 & 1 & 2 & 3.5 & 3.5 \\
7629 & 27 & 27 & 0 & -1 & 0 & 1 & -1 \\
7936 & 28 & 24 & 4 & 1 & 4 & 4.5 & 4.5 \\
7828 & 30 & 31 & -1 & -1 & 1 & 2.5 & -2.5 \\
2194 & 25 & 23 & 2 & 1 & 2 & 3.5 & 3.5 \\
\hline Note: Positive sum $=18.5$, Negative sum $=-3.5$, Test statistic $=3.5$, Critical value $=2$. Higher scores in the post section are indicative of higher QOL.
\end{tabular}

Table 4

WHOQOL-BREF Domain 2: Psychological Raw Scores for Comparison of Quality of Life Pre/Post AAT

\begin{tabular}{cccccccc}
\hline Participant & Pre & Post & Difference & Positive & Diff & Rank & Signed Rank \\
\hline 4689 & 25 & 21 & 4 & 1 & 4 & 4.5 & 4.5 \\
1045 & 23 & 21 & 2 & 1 & 2 & 2.5 & 2.5 \\
6932 & 26 & 25 & 1 & 1 & 1 & 1 & 1 \\
7629 & 21 & 19 & 2 & 1 & 2 & 2.5 & 2.5 \\
7936 & 23 & 26 & -3 & -1 & 3 & 3 & -3 \\
7828 & 20 & 25 & -5 & -1 & 5 & 5 & -5 \\
2194 & 18 & 14 & 4 & 1 & 4 & 4.5 & 4.5 \\
\hline Note: Positive sum $=15$, Negative sum $=-8$, Test statistic $=8$, Critical value $=2$. Higher scores in the post section are indicative of higher QOL.
\end{tabular}


Table 5

WHOQOL-BREF Domain 3: Social Relationship Raw Scores for Comparison of Quality of Life Pre/Post AAT

\begin{tabular}{cccccccc}
\hline Participant & Pre & Post & Difference & Positive & Diff & Rank & Signed Rank \\
\hline 4689 & 8 & 5 & 3 & 1 & 3 & 4 & 4 \\
1045 & 10 & 8 & 2 & 1 & 2 & 3.5 & 3.5 \\
6932 & 7 & 6 & 1 & 1 & 1 & 2.5 & 2.5 \\
7629 & 8 & 7 & 1 & 1 & 1 & 2.5 & 2.5 \\
7936 & 8 & 7 & 1 & 1 & 1 & 2.5 & 2.5 \\
7828 & 6 & 8 & -2 & -1 & 2 & 3.5 & -3.5 \\
2194 & 6 & 6 & 0 & -1 & 0 & 1 & -1 \\
\hline Note: Positive sum $=$ & 15, Negative sum $=-4.5$, Test statistic $=$ 4.5, Critical value= & 2. Higher scores in the post section are indicative of higher QOL.
\end{tabular}

Table 6

WHOQOL-BREF Domain 4: Environment Raw Scores for Comparison of Quality of Life Pre/Post AAT

\begin{tabular}{cccccccc}
\hline Participant & Pre & Post & Difference & Positive & Diff & Rank & Signed Rank \\
\hline 4689 & 33 & 27 & 6 & 1 & 6 & 5 & 5 \\
1045 & 36 & 32 & 4 & 1 & 4 & 4 & 4 \\
6932 & 26 & 26 & 0 & -1 & 0 & 1 & -1 \\
7629 & 27 & 25 & 2 & 1 & 2 & 3 & 3 \\
7936 & 34 & 35 & -1 & -1 & 1 & 2 & -2 \\
7828 & 20 & 31 & -11 & -1 & 11 & 7 & -7 \\
2194 & 21 & 14 & 7 & 1 & 7 & 6 & 6
\end{tabular}

Note: Positive sum=18, Negative sum $=-10$, Test statistic $=10$, Critical value $=2$. Higher scores in the post section are indicative of higher QOL.

In table 7, blood pressure recordings were measured using the Wilcoxon signed-rank test. The test statistic for the blood pressure recordings was 0 which means the data was significant as this score is less than the critical value of 2. In this case, the null hypothesis is rejected, and the alternative hypothesis was accepted. Thus, indicating AAT does have an impact on blood pressure pre and post sessions. Blood pressure medications were recorded for the participants who had high blood pressure on file. The following medications related to high blood pressure were recorded from medical records metoprolol extended release, amlodipine, carvedilol, lisinopril, and losartan. Two participants did not have any blood pressure medications listed on their medical record. For one participant without high blood pressure, their pre and post scores 
dropped during the presence of the therapy dog influencing their blood pressure. Participants who were on high blood pressure medication were observed to have a decrease in blood pressure recording for most sessions. Another factor assumed to effect blood pressure was an increase in excitement during the interaction with the therapy dog which could raise blood pressure.

Table 7

Blood Pressure Results Pre and Post AAT

\begin{tabular}{cccccccc}
\hline Participant & Pre & Post & Difference & Positive & Diff & Rank & Signed Rank \\
\hline 4689 & 3 & 3 & 0 & 1 & 0 & 1 & 1 \\
1045 & 1 & 1 & 0 & 1 & 0 & 1 & 1 \\
6932 & 3 & 2 & 1 & 1 & 1 & 4 & 4 \\
7629 & 4 & 3 & 1 & 1 & 1 & 4 & 4 \\
7936 & 3 & 4 & -1 & 1 & 1 & 4 & 4 \\
7828 & 3 & 3 & 0 & 1 & 0 & 1 & 1 \\
2194 & 3 & 1 & 2 & 1 & 2 & 7 & 7 \\
\hline
\end{tabular}

Note:1-Normal, 2-Elevated, 3-Hypertension Stage 1, 4-Hypertension Stage 2. Positive sum=22, Negative sum=0, Test statistic=0, Critical value $=2$

Clinical observations were observed and monitored throughout each session. Each participant remained engaged in the session when the therapy dog was present. Agitated behaviors observed during administration of assessments for certain participants were absent when interacting with the therapy dog. The mild dementia group was able to associate the researcher with the therapy dog and the day of the week it was when researcher was present. Participants asked the dog handler questions pertaining to the dog including the dog's name, age, breed, and what the dog eats. Participants expressed gratitude for the opportunity to interact with the therapy dog. Participants often stated the following phrases "the dog is so pretty" "I love her" "we are so blessed you are here". The participants reminisce about previous dogs they had owned in their lifetime and how much they had loved them. The participants carried on conversations amongst themselves and with the dog handler and researcher throughout the hour session. One of the participants enjoyed singing while brushing the dog and they were observed 3-4 times during most sessions to sing to the dog. Another participant requested to leave the 
session to use the restroom which the researcher assumed was an escape tactic, however, the participant was able to recall they were in the session and returned. It was assumed the participant valued their time in the session and wanted to be there to interact with the therapy dog. During the third session, one participant expressed sadness of missing their mother and wanting to leave, however, when the therapy dog was brought over, they became happy and engaged with the dog. Participants had the opportunity for the therapy dog to lay on their lap which each participant enjoyed as the sessions went on. Three participants were apprehensive the first week about having the dog sit on their lap, however, a towel was placed on their lap during the next session and then they wanted the dog to sit on their lap. When each participant had the dog on their lap, they were observed to be smiling, hugging, kissing, and petting the dog. Participants were observed making noises, tapping, and reaching to get the dog's attention. When participants were asked what they would change about the study, they reported they wished the sessions were longer.

\section{Summary}

Self-reports did not yield statistically significant data making the use of animal therapy questionable to decrease depression and improve quality of life. Small sample sizes and a short duration of this study are major factors impacting the collection of statistically significant data. However, clinical observations observed over the 6 sessions in the study noted positive behaviors demonstrated when the therapy dog was present. Further studies should be conducted with a focus on how the presence of a therapy dog influences depression and quality of life in patients with dementia living in a nursing home. These changes in behavior should be assessed with an alternative assessment from self-reports, as it can be difficult to determine if the information being collected is an accurate depiction of their life and how they feel. 


\section{Chapter 5: Summary}

\section{Introduction}

Research regarding the effectiveness of AAT with people who have dementia residing in a nursing home remains limited. Reported evidence lacks significance due to the progressive nature of the disease impacting cognition. Additionally, the lack of larger sample sizes plays a major factor in obtaining statistically significant evidence. It is assumed cognitive decline negatively impacts the use of self-report questionnaires with this population and alternative measures should be considered. Clinical observations demonstrated positive human experience outcomes with the therapy dog noting laughter, social engagement with other participants, and reduced agitated behaviors. Further studies should be conducted to determine the significance of AAT with this population. More than one facility should be included in the study to increase sample sizes and generalizability. Also, focusing on physiological changes could potentially provide statistically significant data since it focuses on changes happening within the body. Researchers could focus on hormonal changes taking place in the body when interacting with a therapy dog such as production of endorphins, serotonin, norepinephrine, epinephrine, and cortisol levels (Beetz et al., 2012). This would help to determine if there are any internal changes taking place in the body that could provide evidence to the use of AAT.

\section{Summary of the Study's Results}

The BDI and WHOQOL-BREF self-report questionnaires did not produce statistically significant data. The statistical tests for both questionnaires were above the critical value which means the null hypothesis was then accepted. Other factors that could have skewed this data include a small sample size, uneven distribution of the two groups, utilization of self-report questionnaires, understanding of questions being asked, being able to accurately reflect on how 
they feel, recalling information over the past two weeks, time of day the questionnaires were administered, and the type of day the person was having.

Nonetheless, blood pressure results had a test statistic below the critical value in which the alternative hypothesis is then accepted. Thus, indicating AAT positively influenced blood pressure for patients who have dementia. It can be assumed the presence of a dog can help to reduce blood pressure and result in a calming effect.

Clinical observations made during this study included the presence of laughter, enjoyment as witnessed by singing, smiling, initiating conversations with other, and lack of agitation presented in previously observed participants. Based on the information collected regarding behaviors witnessed during the sessions, it can be inferred a therapy dog helps to increase positive behaviors in patients with dementia. Additionally, family members of other residents living in the nursing home who were not in the study also were interested in learning how their mom/dad could get involved.

\section{Limitations}

Limitations of this project included a short time frame of 6 weeks, a small sample size of seven people, and data being collected in one facility. The findings collected from this study will not be used as generalizable data for the entire population of dementia.

\section{Delimitations}

This study focused on administering assessments related to depression and quality of life pre/post observations of interactions between the therapy dog and participants with dementia at a Central Florida skilled nursing facility. The study included participants 65 years and older who had mild to moderate dementia with interests in dogs. 


\section{Implications for the Project to the Literature}

This study serves as a basis for the development of future studies to determine the impact AAT has on people with dementia living in a nursing home. It contributes evidence to the database that self-report questionnaires may not be the best way to evaluate information. Also, it stresses the importance of a larger sample size and including more than one facility in the study is important to obtain statistically significant data. Additionally, this study it helps to spark other ideas to collect significant data such as by physiological changes like heart rate, blood pressure, respiration rate, oxygen saturation rates, and hormonal changes. This study will help show other OTs the lack of literature available regarding the use of AAT and OT together. This should encourage others in the OT profession to investigate current literature and how a new study could impact the use of AAT by providing statistically significant evidence. The clinical observations in this study revealed positive behaviors, however, to what significance this has on the participants quality of life and depression remains unknown.

\section{Implications for the project to the Profession}

This study serves as a basis for future studies with an OT focus. While AAT has shown to provide many benefits associated with its use, the lack of evidence still limits its use in interventions. OTs could determine how physiological factors are impacted when completing everyday tasks in the presence of a therapy dog. This would help determine if an animal present helps make tasks easier for residents to complete by being in a better state of mind. This study will also help to initiate future conversations with OT practices to get involved in research regarding AAT use. AAT could serve as a non-traditional intervention to work on tasks such as strengthening or using the dog as a talking point as to why grooming and self-care are important for us to complete daily. The hope is the residents would engage in taking care of themselves the 
way they care for an animal which would hopefully improve their quality of life. Lastly, as studies involving OTs and the use of AAT is lacking, using this study as evidence as to why future studies are imperative to determine its use with this population will hopefully motivate other OTs to conduct a study on AAT involvement.

\section{Implications for the Project to the Population Served and Society}

Although statistical data was not collected regarding depression and quality of life, clinical observations of behaviors determined there is positive outcomes associated with AAT use. The facility this study was conducted at agreed to allow the dog handler and their organization to come back to the facility to provide individual visits with the therapy dog and residents. The participants in the study expressed how much they loved the interaction with therapy dog and would like for them to come back for more. Also, other families in the facility who were requesting private visits for their family member that was not involved in the study will be able to engage with the therapy dog. The participants in this study were examined to initiate conversations with others and even the dog during each session. This shows therapy dogs can have a positive impact for residents who have dementia living in a nursing home by providing happiness, joy, and laughter.

\section{Conclusion}

This study had similar findings as previous studies mentioned in the literature review sections of this paper. The degree to which AAT has an impact on the quality of life and depression in patients who have dementia living in a nursing home remains unclear. While clinical observations of AAT revealed positive interactions and increased social engagement, it is difficult to determine how this impacts the patient's perceived quality of life. Further studies should be conducted regarding the efficacy of AAT use in patients who have dementia. Clinical 
observations of behaviors observed at each session were consistent with laughter, smiling, singing, engagement in conversation, petting, and brushing the dog. A future study could build on how the participants demeanor was during interactions with the therapy dog but include a physiological component. This could be by measuring heart rate, respiration rate, oxygen saturation, and blood pressure continuously throughout the session by having the participant monitored on a screen. This would help to determine how different emotions impact all these factors when engaging with a therapy dog. 


\section{References}

American Occupational Therapy Association. (2019). Dementia and the role of occupational therapy. Retrieved from https://www.aota.org/About-Occupational Therapy/Professionals/PA/Facts/Dementia.aspx

Aarskog, N. K., Hunskär, I., \& Bruik, F. (2019). Animal-assisted interventions with dogs and robotic animals for residents with dementia in nursing homes: A systematic review. Physical \& Occupational therapy in geriatrics, 37(2), 77-93. doi: $10.1080 / 02703181.2019 .1613466$

Alzheimer's Association. (2019). Facts and figures. Retrieved from https://www.alz.org/alzheimers-dementia/facts-figures

Alzheimer's Society. (2016). Risk factors for dementia. Retrieved from https://www.alzheimers.org.uk/sites/default/files/pdf/factsheet_risk_factors_for_dementia .pdf

Baek, M. J., Kim, K., Park, Y. H., \& Kim, S. (2016). The validity and reliability of the MiniMental State Examination-2 for detecting mild cognitive impairment and Alzheimer's disease in a Korean population. PloS one, 11(9). doi:10.1371/journal.pone.0163792

Banks, M. R. \& Banks, W. A. (2002). The effects of animal-assisted therapy on loneliness in an elderly population in long-term care facilities. Journal of Gerontology, 57A(7), M428M432.

Bauer, M., Fetherstonhaugh, D., Haesler, E., Beattie, E., Hill, K. D., \& Poulos, C. J. (2018). The impact of nurse and care staff education on the functional ability and quality of life of people living with dementia in aged care: A systematic review. Nurse Education Today, 67, 27-45. doi: 10.1016/j.nedt.2018.04.019 
Beetz, A., Uvnäs-Moberg, K., Julius, H., \& Kotrschal, K. (2012). Psychosocial and psychophysiological effects of human-animal interactions: the possible role of oxytocin. Frontiers in Psychology, (3), 1-15. doi: 10.3389/fpsyg.2012.00234.

Bernabei, V., De Ronchi, D., La Ferla, T., Moretti, F., Tonelli, L., Ferrari, B., . .Atti, A. R. (2013). Animal-assisted intervention for elderly patients affected by dementia or psychiatric disorders: A review. Journal of Psychiatric Research, 47(6), 762-773. doi: 10.1016/j.psychives.2012.12.014

Bernstein, P. L., Friedmann, E., \& Malaspina, A. (2000). Animal-assisted therapy enhances resident social interaction and initiation in long-term care facilities. Anthrozoos, 13(4), 213-224. doi: $10.2752 / 0892793000786999743$

Brodaty, H. \& Donkin, M. (2009). Family caregivers of people with dementia. Dialogues in Clinical Neuroscience, 11(2), 217-228.

Centers for Disease Control and Prevention. (2003). Evaluation Guide: Developing and using a logic model. Retrieved from https://www.cdc.gov/dhdsp/docs/logic_model.pdf

Centers for Disease Control and Prevention. (2016). Alzheimer Disease. Retrieved from https://www.cdc.gov/nchs/fastats/alzheimers.htm

Centers for Disease Control and Prevention. (2018). Alzheimer's Disease and Related Dementias. Retrieved from https://www.cdc.gov/features/alzheimers-diseasedementia/index.html

Centers for Disease Control and Prevention. (2018). Logic Models. Retrieved from https://www.cdc.gov/eval/logicmodels/index.htm 
Chur-Hansen, A., Stern, C., \& Winefield, H. (2010). Gaps in evidence about companion animals and human health: some suggestions for progress. International Journal of EvidenceBased Healthcare, 8(3), 140-146. doi: 10.1111/j.17441609.2010.00176.x

Cipriani, J., Cooper, M., DiGiovanni, N. M., Litchkoski, A., Nichols, A. L., \& Ramsey, A. (2013). Dog-assisted therapy for residents of long-term care facilities: an evidence-based review with implications for occupational therapy. Physical \& Occupational Therapy in Geriatrics, 31(3), 214-240. doi: 10.3109/02703181.2013.816404

Cole, M. B., \& Tufano, R. (2008). Applied theories in occupational therapy: a practical approach (pp.149-163). Thorofare, NJ: Slack.

De Bellis, A., Bradley, S. L., Xiao, L. D., Belan, I., \& Wallace, T. (2017). Antipsychotic use for behaviours by persons with dementia in residential aged care: the relatives' perspectives. Australian Journal of Advanced Nursing, 35(1), 23-32.

De Rooij, A. HPM., Luijkx, K. G., Declercq, A. G., \& Schols, J. MGA. (2011). Quality of life of residents with dementia in long-term care settings in the Netherlands and Belgium: design of a longitudinal comparative study in traditional nursing homes and small-scale living facilities. BMC Geriatrics, 11(20), 1-9.

Frantz, N. (2015). New analysis shows more than 28 million baby boomers will develop Alzheimer's Disease: will consume nearly 25\% of Medicare spending. Alzheimer's Association. Retrieved from https://www.alz.org/aaic/_downloads/mon-930am-babyboomers.pdf

Fick, K. (1993). The influence of an animal on social interactions of nursing home residents in a group setting. American Journal of Occupational Therapy, 47(6), 529-534. 
García-Batista, Z. E., Guerra-Pena, K., Cano-Vindel, A., Herrera-Martínez, X., \& Medrano, L. A. (2018). Validity and reliability of the Beck Depression Inventory (BD-II) in general and hospital population of Dominican Republic. PLoS ONE, 13(6). doi: 10.1371/journal.pone.0199750

Gately, M. E., \& Trudeau, S. A. (2017). Occupational therapy and advanced dementia: a practitioner survey. Journal of Geriatric Mental Health,4, 48-53. doi: 10.4103/jgmh.jgmh_41_16

Grubbs, B., Artese, A., Schmitt, K., Cormier, E., \& Panton, L. (2016). A pilot study to assess the feasibility of group exercise and animal-assisted therapy in older adults. Journal of Aging and Physical Activity, 24 (2), 322-331. doi: 10.1123/japa.2015-0107

Hu, M., Zhang, P., Leng, M., Li, M., \& Chen, L. (2018). Animal-assisted intervention for individuals with cognitive impairment: a meta-analysis of randomized controlled trials and quasi-randomized controlled trials. Psychiatry Research, 260, 418-427. doi: 10.1016/j.psychres.2017.12.016.

Kanamori, M., Suzuki, M., Yamamoto, M., Kanda, M., Matsui, Y., Kojima, E., . . Oshiro, H. (2001). A day care program and evaluation of animal-assisted therapy (AAT) for the elderly with senile dementia. American Journal of Alzheimer's Disease and Other Dementias, 16 (4), 234-239. doi: 10.1177/1533331750101600409

Kårefjärd, A., \& Nordgren, L. (2018). Effects of dog-assisted intervention on quality of life in nursing home residents with dementia. Scandinavian Journal of Occupational Therapy, 26(6), 433-440. doi: 10.1080/11038128.2018.1467486 
Klimova, B., Toman, J., \& Kuca, K. (2019). Effectiveness of the dog therapy for patients with dementia- a systematic review. BMC Psychiatry, 19, 276. 1-10. doi: 10.1186/s12888-0192245-X

López-Cepero Borrego, J., Rodríguez Franco, L., Prea Mediavilla, M., Blanco Piñero, N., Tejada Roldán, A., \& Blanco Picabia, A. (2014). Animal-assisted interventions: review of current status and future challenges. International Journal of Psychology and Psychological Therapy, 14 (1), 85-101.

Lucas-Carrasco, R., Skevington, S. M., Gómez-Benito, J., Rejas, J., \& March, J. (2011). Using the WHOQOL-BREF in persons with dementia: a validation study. Alzheimer Disease \& Associated Disorders, 25(4), 345-351. doi: 10.1097/WAD.0b013e31820bc98b

Lundqvist, M., Carlsson, P., Sjödahl, R., Theodorsson, E., \& Levin, L. Å. (2017). Patient benefit of dog-assisted intervention in health care; a systematic review. BMC Complementary and Alternative Medicine, 17(358), 1-12. doi: 10.1186/s12906-017-1844-7

Makai, P., Beckebans, F., Van Exel, J., \& Brouwer, W. B. F. (2014). Quality of life of nursing home residents with dementia: validation of the German version of the ICECAP-O. PLOS One, 9(3). 1-10. doi: 10.1371/journal.pone.0092016

Marx, M. S., Cohen-Mansfield, J., Regier, N. G., Dakheel-Ali, M., Srihari, A., \& Thein, K. (2010). The impact of different dog-related stimuli on engagement of persons with dementia. American Journal of Alzheimers Disease and Other Dementias, 25(1), 37-45. doi: $10.1177 / 1533317508326976$

Mendes, A. (2019). Alternatives to (antipsychotic) medication in people with dementia. British Journal of Nursing, 28(8) 534. 
Motomura, N., Yagi, T., \& Ohyama, H. (2004). Animal assisted therapy for people with dementia. Psychogeriatrics, 4, 40-42.

Muñoz Lasa, S. \& Franchignoni, F. (2008). The role of animal-assisted therapy in physical and rehabilitation medicine. European Journal of Physical and Rehabilitation Medicine, 44, $1-2$.

Nordgren, L. \& Engström, G. (2014). Effects of dog-assisted intervention on behavioral and psychological symptoms of dementia. Art \& Science, 26(3), 31-38.

Pope, W. S., Hunt, C., \& Ellison, K. (2016). Animal-assisted therapy for elderly residents of a skilled nursing facility. Journal of Nursing Education and Practice, 6 (9), 56-62. doi: 10.5430/jnep.v6n9p56

Portney L. G. \& Watkins, M. P. (2015). Glossary. In Foundation of clinical research: Applications to practice ( $3^{\text {rd }}$ ed, pp.873). Philadelphia, PA: F.A. Davis Company.

Psychology wiki. (2019). Psychosocial factors. Retrieved from https://psychology.wikia.org/wiki/Psychosocial_factors

Sánchez-Valdeón, L., Fernández-Martínez, E., Loma-Ramos, S., López-Alonso, A. I., Bayón Darkistade, E., \& Ladera, V. (2019). Canine-assisted therapy and quality of life in people with Alzheimer-type dementia: Pilot study. Frontiers in Psychology, 10, 1-6. doi: 10.3389/fpsyg.2019.01332

Sellers, D. M. (2006). The evaluation of an animal assisted therapy intervention for elders with dementia in long-term care. Activities, Adaptation \& Aging, 30(1), 61-77. doi: 10.1300/J016v30n01_04

Shirley Ryan Ability Lab. (2014). WHO quality of life-BREF (WHOQOL-BREF). Retrieved from https://www.sralab.org/rehabilitation-measures/who-quality-life-bref-whoqol-bref 
Smallfield, S. (2017). Dementia and the role occupational therapy. American Occupational Therapy Association. Retrieved from https://www.aota.org/About-OccupationalTherapy/Professionals/PA/Facts/Dementia.aspx

Smith, M., Buckwalter, K. C., Kang, H., Ellingrod, V., \& Schultz, S. K. (2008). Dementia care in assisted living: needs and challenges. Issues Mental Health Nursing, 29(8), 817-837. doi: $1080 / 01612840802182839$

State Affairs Group. (2019). Occupational therapy profession-scope of practice definitions. Retrieved from https://www.aota.org/ /media/Corporate/Files/Advocacy/Licensure/StateRegs/Scope-ofPractice-Chart-2019.pdf

Statistical Solutions. (2019). Beck depression inventory (BDI). Retrieved from https://www.statisticssolutions.com/beck-depression-inventory-bdi/

Tombaugh, T. N. \& McIntyre, N.J. (1992). The mini-mental state examination: a comprehensive review. Journal of the American Geriatrics Society, 40(9), 922-935. doi: 10.1111/j.15325415-1992.tb01992.x

Tournier, I., Vives, M.F., Postal, V. (2017). Animal-assisted intervention in dementia: effects on neuropsychiatric symptoms and on caregivers' distress perceptions. Swiss Journal of Psychology, 76(2), 51-58. doi: 10.1024/1421-0185/a000191.

University of Minnesota. (2019). What is a logic model?. Retrieved from https://www.cyfar.org/what-logic-model

Velde, B.P., Cipriani, J., \& Fisher, G. (2005). Resident and therapist views of animal-assisted therapy: implications for occupational therapy practice. Australian Occupational Therapy Journal, 52, 43-50. doi: 10.1111/j.1440-1630.2004.00442.x 
Wagle, A. C., Ho, L. W., Wagle, S. A., \& Berrios, G. E. (2000). Psychometric behavior of BDI in Alzheimer's disease patients with depression. International Journal of Geriatric Psychiatry, 15(1), 63-69.

Wetzels, R. B., Zuidema, S. U., De Jonghe, J. F. M., Verhey, F. R. J. \& Koopmans, R. T. C. M. (2010). Determinants of quality of life in nursing home residents with dementia. Dementia Geriatric Cognitive Disorders, 29(3), 189-197. doi: 10.1159/000280437

Williams, E., \& Jenkins, R. (2008). Dog visitation therapy in dementia care: a literature review. Nursing Older People, 20(8), 31-35. doi: 10.7748/nop2008.10.20.8.31.c6808

Winkle, M. Y., \& Ni, K. (2019). Animal-assisted intervention 101. Retrieved from https://www.aota.org/publications-news/otp/archive/2019/animal-assisted.aspx

World Health Organization. (2019). WHOQOL: measuring quality of life. Retrieved from https://www.who.int/healthinfo/survey/whoqol-qualityoflife/en/index2.html

Zafra-Tanaka, J. H., Pacheco-Barrios, K., Tellez, W. A., \& Taype-Rondan, A. (2019). Effects of dog-assisted therapy in adult with dementia: a systematic review and meta-analysis. $B M C$ Psychiatry, 19(41), 1-10. doi: 10.1186/s12888-018-2009-z 